\title{
Molecular alterations of Ras-Raf-mitogen-activated protein kinase and phosphatidylinositol 3-kinase-Akt signaling pathways in colorectal cancers from a tertiary hospital at Kuala Lumpur, Malaysia
}

\begin{abstract}
Molecular alterations in KRAS, BRAF, PIK3CA, and PTEN have been implicated in designing targeted therapy for colorectal cancer (CRC). The present study aimed to determine the status of these molecular alterations in Malaysian CRCs as such data are not available in the literature. We investigated the mutations of KRAS, BRAF, and PTEN, the gene amplification of PIK3CA, and the protein expression of PTEN and phosphatidylinositol 3kinase (PI3K) catalytic subunit $(\mathrm{p} 110 \alpha)$ by direct DNA sequencing, quantitative real-time PCR, and immunohistochemistry, respectively, in 49 CRC samples. The frequency of KRAS (codons 12, 13, and 61), BRAF (V600E), and PTEN mutations, and PIK3CA amplification was $25.0 \%$ (11/44), 2.3\% (1/43), $0.0 \%$ (0/43), and 76.7\% (33/43), respectively. Immunohistochemical staining demonstrated loss of PTEN protein in 54.5\% (24/44) of CRCs and no significant difference in PI3K p110 $\alpha$ expression between CRCs and the adjacent normal colonic mucosa $(\mathrm{p}=0.380)$. PIK3CA amplification was not associated with PI3K p1 $10 \alpha$ expression level, but associated with male cases $(100 \%$ of male cases vs $56 \%$ of female cases harbored amplified PIK3CA, $p=0.002)$. PI3K $p 110 \alpha$ expression was significantly higher $(\mathrm{p}=0.041)$ in poorly/moderately differentiated carcinoma compared with well-differentiated carcinoma. KRAS mutation, PIK3CA amplification, PTEN loss, and PI3K p110 $\alpha$ expression did not correlate with Akt phosphorylation or Ki-67 expression. KRAS mutation, PIK3CA amplification, and PTEN loss were not mutually exclusive. This is the first report on CRC in Malaysia showing comparable frequency of KRAS mutation and PTEN loss, lower BRAF mutation rate, higher PIK3CA amplification frequency, and rare PTEN mutation, as compared with published reports.
\end{abstract}

Keyword: KRAS; BRAF; PTEN; PIK3CA; Akt; Colorectal cancer 